\title{
Interactive comment on "GIR v1.0.0: a generalised impulse-response model for climate uncertainty and future scenario exploration" by Nicholas James Leach et al.
}

Nicholas James Leach et al.

nicholas.leach@stx.ox.ac.uk

Received and published: 2 August 2020

Please find our response attached.

Please also note the supplement to this comment:

https://gmd.copernicus.org/preprints/gmd-2019-379/gmd-2019-379-AC2-

supplement.pdf 